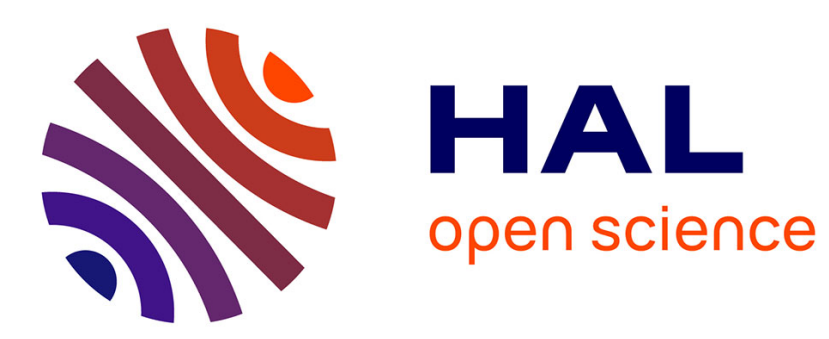

\title{
L'analyse d'une institution: Le Registre du commerce et les étrangers dans l'entre-deux-guerres
}

Claire Zalc

\section{To cite this version:}

Claire Zalc. L'analyse d'une institution: Le Registre du commerce et les étrangers dans l'entre-deuxguerres. Genèses. Sciences sociales et histoire, 1998, 31 (1), pp.99 - 118. 10.3406/genes.1998.1512 . hal-03416607

\section{HAL Id: hal-03416607 \\ https://hal.science/hal-03416607}

Submitted on 5 Nov 2021

HAL is a multi-disciplinary open access archive for the deposit and dissemination of scientific research documents, whether they are published or not. The documents may come from teaching and research institutions in France or abroad, or from public or private research centers.
L'archive ouverte pluridisciplinaire HAL, est destinée au dépôt et à la diffusion de documents scientifiques de niveau recherche, publiés ou non, émanant des établissements d'enseignement et de recherche français ou étrangers, des laboratoires publics ou privés. 


\section{L'analyse d'une institution: Le Registre du commerce et les} étrangers dans l'entre-deux-guerres

Claire Zalc

\section{Citer ce document / Cite this document :}

Zalc Claire. L'analyse d'une institution: Le Registre du commerce et les étrangers dans l'entre-deux-guerres. In: Genèses, 31, 1998. Femme, famille, individu. pp. 99-118;

doi : https://doi.org/10.3406/genes.1998.1512

https://www.persee.fr/doc/genes_1155-3219_1998_num_31_1_1512

Fichier pdf généré le 14/05/2018 


\title{
Résumé
}

- Claire Zalc: Le Registre du commerce et les étrangers dans l'entre-deux-guerres. Histoire d'une source La présentation d'une source historique mal connue, le Registre du; commerce, amène: ai questionner les modalités d'utilisation par le chercheur des renseignements figurant dans un document officiel et, plus généralement, soulève le problème des conditions de production - des catégories administratives. Pourquoi faire figurer la nationalité dans un document économique ? Analyser l'histoire des débats relatifs < au Registre du commerce, dans l'entre-deux-guerres ne relève pas seulement d'une exigence méthodologique, c'est aussi montrer la place, que les étrangers tiennent dans la rhétorique légitimant l'identification étatique des commerçants. L'exploitation ; statistique de la source se trouve alors enrichie par de nouvelles problématiques d'approche, en particulier la questiondu nom du commerce.

\begin{abstract}
The Trade Register and foreigners between the wars. History of a source. Introducing the Trade Register, a little-known historical source, leads to questioning: the way researchers use the information, contained in official documents and, more generally, raises the problem of the conditions under which administrative categories have been developed. Why should nationality appear in an economic document? Analysing the history of the debates relating to the Trade Register between the wars is not only necessary from a methodological standpoint, it also demonstrates the role played by foreigners in the rhetoric used to legitimate state identification of i shopkeepers. Statistical use . of the source is therefore enriched by new problems of approach; especially the question of the trade name.
\end{abstract}




\section{L'analyse}

d'une institution:

Le Registre

du commerce

et les étrangers dans

\section{l'entre-deux-guerres}

\section{Claire Zalc}

\section{$\longrightarrow$}

* Cet article est la version remaniée d une communication au colloque Sciences sociales, filat el sociétí organisé les 8. 9 et 10 septembre 1997 à Rio de Janeiro. Brésil. par le Musée national de l'Université fédérale de Rio et le département de sciences sociales de l'École normale supéricure.

1. Procès-verbal de la séance du 2.3 novembre 1938. Archives de la Chambre de commerce et d'industrie de Paris (AC'CIP) cote III.4.44 (2).

2. Norberl Elias par lui-méme, entretiens biographiques avec Norbert Llias, Paris, Fayard. 1991. p. 67.

3. L’exercice de la profession médicale est ainsi interdit aux médecins étrangers par la loj du 21 avril 1933 et à tout Français naturalisé depuis moins de dix ans selon la loi du 26 juillet 1935 dite loi A rmbruster, voir Jean-Charles Bonnet. Lees pouvoirs publics et limmigration dans l'entredeux-guerres, thèse de troisieme cycle d'Histoire.

Université de Lyon. Lyon. Publications du Centre Pjerre Léon. 1974.
"Lorsqu'il s'agit d'un État commerçant, il ne veut pas d'étrangers. Il est impossible à un étranger de tenir un café-bureau de tabac; et quand l'État fait faire des travaux qu'il donne à des entreprises, il entend fixer le nombre d'étrangers qui y travaillent. Ce que l'État fait, ce que vous acceptez pour l'État, vous ne voudriez pas l'accepter pour nous? L'État,

c'est nous. Je ne vois pas quelle est la différence.»

(Marcel Bagnaud, membre de la Commission du Travail et des Questions sociales de la Chambre de commerce de Paris) ${ }^{1}$

La recherche emprunte parfois des chemins peu balisés et c'est presque fortuitement que j'ai, pour la première fois, eu affaire au Registre du commerce du département de la Seine devenu, depuis, l'une des sources essentielles de mon travail*. L'anecdote est amusante. J'étais à la recherche de traces archivistiques du passage de Norbert Elias à Paris. Comme il le raconte en effet dans ses entretiens autobiographiques, Elias fuit l'Allemagne nazie à l'automne 1933 pour la France où il cherche pendant quelques mois à trouver du travail dans une université. Mais son titre prestigieux de professeur à l'Université d'Heidelberg n'y fait rien. Les portes des institutions universitaires se ferment. "Ce fut une période difficile pour moi, la seule durant laquelle j'aie jamais eu à souffrir de la faim, parce que je n'avais plus d'argent. Cela ne m'a pas fait perdre courage pour autant. On vivait au jour le jour, tout simplement, et l'on gardait l'espoir tant qu'on avait encore de l'argent. Je me souviens encore des deux ou trois jours où je me suis trouvé dans l'impossibilité absoluc de me procurer de l'argent; dans un café, j’ai prié une amic qui était assise à côté de moi de me commander un café et un sandwich $»^{2}$. À l'époque en effet, le marché du travail se barricade devant la crise. Lois et décrets de «protection de la main d'œuvre nationale» viennent limiter l'emploi d'étrangers dans chaque secteur industriel, voire parfois interdire l'accès de certaines professions aux étrangers ${ }^{3}$. Pour Elias, comme pour les quelques quarante mille réfugiés en provenance du Troisième Reich, la situation se révèle bien vite désespé- 


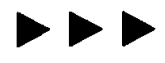

4. Hans Sahl, Die Wenigen und die Vielen-Roman einer Zeit, Francfort, Fischer, 1989.

5. Ce document est reproduit pp. 102-103.

6. L'étude de Jean-Charles Bonnet concernant la région lyonnaise fait figure d'étude pionnière sur cette source. «Étude des petits commerçants étrangers dans l'agglomération lyonnaise (1919-1939) à partir du Registre de commerce ". Bulletin du Centre d'histoire économique et sociale de la région lyonnaise, $n^{\circ} 1,1975$, pp. 1-41.

7. François Gresle montre ainsi la naissance progressive du «groupe statutaire » des indépendants, "La notion de classe moyenne indépendante. Un bilan des travaux ", Vingtième siècle, $\mathrm{n}^{5}$ 37. 1993, pp. 35-43.

81. Le décret du 9 août 1953 qui remanie profondément l'institution explique que, pour la période 1920-1954, le Registre du commerce a été versé aux Archives de la Ville de Paris par le Tribunal de commerce.

9. À compter du 16 juillet 1920, tous les commerçants exerçant dans le département de la Seine sont tenus de s inscrire au Registre du commerce et on compte ainsi près de 300 volumes consacrés aux seules années 19201921.

10. Le nantissement est un contrat par lequel un débiteur remet fictivement un bien à son créancier pour sûreté de sa dette. La loi du 27 mars 1909 prévoit la possibilité de donner un nantissement sur un fonds de commerce sans qu'il y ait dépossession du propriétaire du fonds. rée. Les économies réunies lors de départs très souvent précipités sont rapidement épuisées. Trouver un emploi salarié devient de plus en plus difficile et pour la plupart, la seule solution pour survivre consiste à se lancer dans le petit commerce. Les romans de l'émigration allemande se font l'écho de ces situations vécues comme déclassantes: "Certains écrivent, d'autres ont investi leur dernier sou dans une blanchisserie, ou une agence de photographie, font des gâteaux ou vendent des saucisses qu'ils colportent de maison en maison. Voyez là-bas ce petit homme craintif au lorgnon noir? C'était autrefois un gynécologue berlinois réputé. Aujourd'hui, il parcourt Paris avec sa sacoche et vend des fleurs artificielles $»^{4}$. Norbert Elias n'échappe pas à la règle: il raconte avoir ouvert un atelier de jouets, acheté quelques machines et tenté de vendre pantins et autres objets en bois.

C'est en suivant cette piste que j'ai localisé l'immatriculation au Registre du commerce du département de la Seine, le 20 avril 1934, d'un établissement dénommé «Les Ateliers Norbert », spécialisé dans le modelage de bois, les jouets et les articles pour cadeaux, 34, rue de l'Abbé Groult, dans le $15^{\mathrm{e}}$ arrondissement de Paris, et possédé par Norbert Elias ${ }^{5}$. Ce document de nature anecdotique m'a conduit vers une source jusqu'ici quasiment inexploitée pour aborder l'histoire des petits commerçants étrangers dans l'entre-deux-guerres ${ }^{6}$. Mais avant de se pencher sur les données fournies par le Registre du commerce, il semblait nécessaire d'en faire l'histoire et de s’interroger sur les présupposés idéologiques s'insinuant derrière les catégories administratives qui le composent, afin de comprendre les déterminations engendrées par l'utilisation de cette source.

En effet, l'institution d'un registre du commerce en 1919 n'est pas neutre et nous renseigne sur la mise en place des modalités de contrôle étatique de la société, à une époque 
où l'intervention de l'État dans le monde du travail devient de plus en plus prégnante. De plus, il paraît particulièrement intéressant d'étudier les processus d'identification étatiques dans un secteur du monde du travail traditionnellement perçu comme méfiant, voire hostile à la puissance publique. Or les travailleurs indépendants, soumis à un impôt spécifique, la patente, échappent au contrôle social de l'État puisqu'ils ne bénéficient pas des premières lois sociales, réservées aux seuls salariés jusqu'en 1932 où un décret organise les Caisses de secours aux travailleurs indépendants chômeurs 7 . Comment, dès lors, imposer l'idée d'un registre du commerce? En arguant d'une nécessaire surveillance des petits commerçants étrangers. L'identification des étrangers apparaît ainsi comme un procédé majeur de légitimation de la création du Registre dans les discours des pouvoirs publics. En 1938, le Registre du commerce ne suffit plus. Les étrangers qui désirent s'installer comme commerçants sont contraints d'obtenir une "carte de commerçant étranger». Le cloisonnement national touche l'un des secteurs-refuge d'emploi de main d'œuvre étrangère. N'est-il pas alors possible d'interpréter cette mesure comme l'héritière des modalités successives d'identification et de contrôle mises en places depuis la première guerre mondiale?

En étudiant les débats relatifs à la création puis aux modifications du Registre de commerce, il nous faudra décrire le processus qui mène à l'institution d'une "carte de commerçant étranger». Enfin, il sera nécessaire de réfléchir aux incidences de l'identification étatique sur la construction de l'identité sociale, voire personnelle de l'individu et, plus précisément, de saisir comment les petits commerçants perçoivent les catégories administratives. Or les informations contenues dans le Registre de commerce permettent d'approcher quantitativement les conséquences des désignations administratives sur les individus.

\section{Un document administratif}

\section{Description}

Plus de mille livres dont la reliure s'effrite à force d'être manipulée par les services spécialisés des Archives de la Ville de Paris, chargés de répondre aux très nombreux commerçants qui ont besoin de fournir la preuve de leur immatriculation au registre pour toucher leur retraite, le registre du commerce du département de la Seine impressionne à première vue par son importance. Chaque volume in-quarto formant le Registre analytique contient deux-cent-cinquante pages sur chacune desquelles deux à quinze individus ont été inscrits, par le greffier chargé de noter scrupuleusement l'ensemble des informations déclarées lors de l'immatriculation. Plus d'un million d'inscriptions ont été enregistrées entre 1920 et 1954, inscriptions qui font également l'objet, chacune, d'un recensement sous la forme de petites fiches cartonnées, rangées par ordre alphabétique et permettant de retrouver, à partir du nom du commerçant, le volume correspondant à la date de son immatriculation ${ }^{8}$. Répertoire officiel des personnes physiques et morales qui exercent une activité commerciale, le Registre du commerce contient des informations sur les établissements commerciaux.

Il fournit des renseignements sur la date d'immatriculation et la date d'ouverture du commerce (qui ne sont pas toujours identiques, en particulier lors de la mise en place du Registre en juillet 1920 supposé recenser l'ensemble des petits commerces ouverts alors à Paris et dans sa banlieue ${ }^{9}$ ), et éventuellement la date de la radiation du commerce avec, le cas échéant, mention de la raison de fermeture (décès, vente, cessation...). Figurent également le nom, l'adresse et l'objet du commerce. Enfin la présence d'associés, l'adresse de l'établissement précédemment occupé, les opérations financières effectuées (nantissements ${ }^{10}$ ) et les faillites mais aussi le dépôt de 
$28 \div$

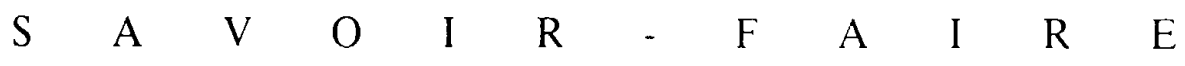

Illustration non autorisée à la diffusion

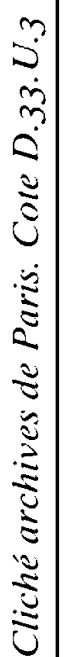




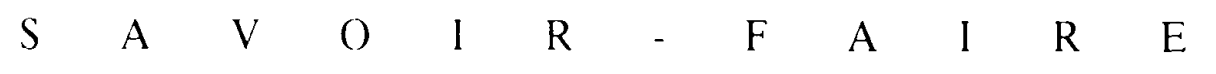

brevets d'invention ou de marques de fabrique sont mentionnés lorsqu ills existent.

La deuxième catégorie d'informations porte sur la personne du commerçant. ses nom et prénom. date de naissance et lieu de naissance (ce renseignement est souvent très précis - à l'échelle de la commune avec mention du département ou du pays d'origine), son régime matrimonial mais surtout, sa nationalité. Et le document indique parfois même la date d’arrivée en France. comme c'est le cas pour Elias où l'on peut lire, sous la nationalité, la mention «24-10-33». Cette information figure systématiquement à partir du milieu des années trente. Enfin. le Registre du commerce présente l'intérêt d'être une source dynamique puisque certains changements de situation y sont notés. Ainsi d'un mariage ou d'un divorce. d'un changement d'adresse. d'un nouveau nom du commerce ou encore d'une liquidation judiciaire. La mention «aryen» est fréquemment rajoutée. au stylo rouge, sous la nationalité du commerçant pendant la Seconde Guerre mondiale.

\section{La déclaration d'identité}

D'aucuns pourront qualifier cette source de "pauvre» en ce qu'elle présente l'aridité des documents administratifs destinés à l'identification et à l'enregistrement. Et pourtant, derrière chaque ligne, semble se cacher une histoire individuelle dont il est possible de suivre les élapes chronologiques. Cette richesse tient en particulier aux modalités spécifiques de l'immatriculation au Registre. L'enregistrement dans la plupart des documents officiels est le produit de linteraction entre le fonctionnaire et l'intéressé. Lors des recensements de la population par exemple, l'agent recenseur chargé de recueillir la déclaration dispose d'une marge de manœuvre relativement importante pour interpréter l'information et. souvent, la rationalise selon des critères précisés dans les directives préfectorales. Or l'origi- 
nalité du Registre du commerce tient aux procédures d'immatriculation: lors de l'inscription, le requérant remet au greffier une déclaration en double exemplaire signée de lui. Le greffier recopie strictement sur le Registre du commerce le contenu de cette déclaration et remet au requérant un des deux exemplaires au bas duquel il certifie en avoir opéré la copie. L'article 14 de la loi du 18 mars 1919, qui institue le Registre du commerce en France, prétend préserver la liberté du déclarant en précisant «que le greffier n'a aucun pouvoir d'appréciation sur les déclarations qui lui sont faites, sauf dans le cas où celles-ci ne contiendraient pas toutes les mentions prescrites par la loi ». Au contraire de l'agent recenseur qui a la possibilité de modifier les informations recueillies puisqu'il est chargé de les inscrire lui-même sur les listes nominatives du recensement en les codifiant selon certaines normes, le greffier du Tribunal de commerce doit se limiter à recopier telle quelle la déclaration du commerçant. Cette dernière n'est pas le fruit d'une négociation entre le greffier et le déclarant, mais plutôt le produit d'une autocodification de l'identité à l'intérieur de cadres imposés.

Parfois la source reflète d'ailleurs les décalages possibles entre ces cadres imposés et la définition de l'individu, lorsque les renseignements requis semblent ne pas correspondre aux schèmes de perception de l'individu. On trouve par exemple, au fil des pages, des indications étonnantes de nationalité ("géorgien grec», «brésilien arménien») qui reflètent le décalage entre la catégorie «nationalité» d'un document administratif et la déclaration individuelle quant à son appartenance nationale.

11. Ce système social dit millet est décrit par Annie Benveniste, Le Bosphore à la Roquette, la communauté judéo-espagnole à Paris (1914-1990), Paris, L'Harmattan, 1989 , p. 24.

12. Marguerite Blocaille-Boutelet détaille les différentes ordonnances prises sous le gouvernement de Vichy et qui, notamment, interdisent aux Juifs l'exercice du commerce de détail (ordonnance du 26 avril 1941), "L'aryanisation des biens" ", Le droit antisémite de Vichy, Le Genre humain, $\mathrm{n}^{\circ} 30$ et 31, Paris, Le Seuil, 1996, pp. 243-265.
Ainsi la mention «israélite» retrouvée à plusieurs reprises pour qualifier la nationalité est significative d'une identité nationale vécue sur un mode religieux, propre aux Juifs orientaux originaires de l'Empire ottoman où, jusqu'en 1839, l'État conférait à chaque communauté religieuse non musulmane une autonomie 
juridique ${ }^{11}$. L'intérêt pour le chercheur d'une telle procédure d'enregistrement est important puisqu'elle garantit la proximité entre les informations figurant dans le Registre et les déclarations individuelles.

Cependant, la particularité de cette procédure d'inscription ne doit pas faire croire que la déclaration d'identité du requérant est "libre" de toute assignation identitaire. L'environnement judiciaire de l'immatriculation (qui s'effectue au greffe du Tribunal de commerce) influence certainement pour une part les déclarations. D'ailleurs il faut noter les ambiguités du discours officiel qui, dans le même article de loi prétend préserver la liberté de déclaration et indique que le greffier peut signaler au président du Tribunal de commerce ou au juge chargé de la surveillance du Registre les inexactitudes qui lui paraissent avoir été commises dans les déclarations (article 14 de la loi du 18 mars 1919). Et les fausses déclarations sont passibles de peines au Tribunal correctionnel - amende de cent à deux mille francs, emprisonnement de un à six mois, privation du droit de vote et d'éligibilité pour le Tribunal et la Chambre de commerce (article 19). Pour autant, il ne faut pas sousestimer les déclarations lacunaires, erronées voire fausses. On comprend aisément que les petits commerçants éprouvent quelques réticences à dévoiler aux autorités certaines informations susceptibles de nuire à leurs affaires. La présence du fonctionnaire est matérialisée par le formulaire d'immatriculation et la source reflète la manière dont l'individu s'accommode des cadres administratifs imposés. Enfin, lors du contexte spécifique de la Seconde Guerre mondiale, l'autorité administrative intervient directement dans le registre du commerce comme en témoignent ces tampons dont la régularité typographique tranche avec les déliés de l'écriture manuscrite et qui annoncent, à l'encre rouge, les radiations des commerces suite aux ordonnances d'aryanisation des biens juifs ${ }^{12}$.

\section{Comment analyser des données} administratives?

Dans la perspective d'une recherche sur les petits commerçants étrangers à Paris, le Registre du commerce offre de nombreuses possibilités. Cette source permet tout d'abord de saisir l'ensemble de la population commerçante du département de la Seine dans les débuts de l'entre-deux-guerres. En effet, institué par la loi du 18 mars 1919, le Registre du commerce est ouvert à l'immatriculation le 16 juillet 1920. A cette date, tous les commerçants sont tenus de s'y inscrire dans un délai de six mois. Les volumes du Registre du commerce pour les années 1920-1921 recensent ainsi l'ensemble des commerçants établis dans le département à cette époque soit près de 150000 immatriculations. De plus, la richesse des informations contenues (près de 15 informations par immatriculation) et le caractère évolutif de la déclaration permettent de construire des itinéraires professionnels. Cependant, la masse des données disponibles rend bien évidemment impossible tout traitement exhaustif et nécessite le recours à la méthode du sondage.

L'exploitation du Registre du commerce se doit de suivre un traitement quantitatif. Or si l'histoire sociale, héritière de l'École des annales, a longtemps revendiqué la suprématie des sources statistiques et des méthodes quantitatives, on assiste depuis le début des années quatre-vingt à une remise en cause de ce modèle qui passe par la critique des statistiques et aboutit à un relatif abandon de l'histoire quantitative au profit d'une «nouvelle» histoire culturelle ou politique, ou d'une histoire sociale privilégiant d'autres types de sources, en particulier orales. Que reproche-ton aux statistiques? Il est nécessaire de comprendre les critiques adressées aux méthodes quantitatives pour pouvoir tenter d'y répondre. Récemment, certains ont mis en valeur les limites des approches qui projettent, 
sur l'analyse de la société. des cadres et des modèles trop restreints par rapport à la complexité du réel et narrivent pas à dépasser le cadre descriptif ${ }^{13}$. Les micro-historiens proposent donc de se livrer à l'examen exhaustif de cas individuels. en comparant les différentes sources disponibles, afin de concevoir un modèle d’analyse précédant l'étude quantitative. Or l'existence d'un fichicr nominatif des commerçants immatriculés au Registre du commerce offre la possibilité de procéder à des études plus fines de cas individuels, en comparant les renseignements obtenus dans le Registre avec d'autres sources (dossiers de faillite. patentes, listes nominatives du recensement etc. $)^{14}$. Une étude en cours sur le $19^{\circ}$ arrondissement, grâce au relevé systématique de tous les petits commerçants italiens dans le quartier de la Villette à partir des listes nominatives des recensements des années vingt, a permis de retrouver ces individus et ceux portant le même patronyme dans le Registre du commerce et de mettre en valeur à la fois les

13. Voir Maurizio Gribaudi el Alain Blum. "Des catégories aux liens individuels: l'analyse statistique de l'espace social", Annales ESC, n 6, 1990). pp. 1365-1402.

14. Voir l'étude de Jean-Luc Pinol sur Lyon qui compare les listes nominatives du recensement avec les listes électorales, Espace social et espace politicute, Lyon à l'époque du From populaire. Lyon, Presses universitaires de Lyon. 1980.

15. Judith Rainhorn et Claire Zalc, «Commerce à litalienne. Immigration et activité professionnelle à Paris dans l'entre-deux-guerres". l.e Mouvement social, à paraître.

16. En témoigne létude menée en 1901 par un professeur de droit de l'université de Paris sur les registres du commerce allemand et suisse, étude qui provoque de nombreuses discussions el débats dans les milicux juridiques, ACCIP cote III. 3.20 (3).

17. Exposé des motifs. Projet de loi tendant à la création d'un registre de commerce. n 2592, 17 octobre 1916, p. 2.

18. Voir Jean-Claude Farcy, Les camps de concentration français de la premiere gaterre mondiale (1914-1920), Paris, Anthropos-Economica. 1995, pp. 33-50.

19. Exposé des motifs. Projet de loi tendant à la création d'un registre de commerce. $n$ 2592, 17 octobre 1916. p. 2.

20. Proposition de loi n" 468, Chambre des députés. 22 décembre 1914. logiques familiales à l'wuvre dans l'activité professionnelle et les parcours migratoires des petits commerçants italiens dans la ville ${ }^{15}$.

Le second défaut de l'histoire quantitative relevé par les critiques concerne l'absence fréquente de réflexion sur les catégories utilisées, en particulier lorsqu'il s'agit de cadres pensés par les statisticiens de l'époque et donc produits de schèmes de pensée administratifs, nécessairement orientés. Concernant le Registre du commerce, il convient en particulier de s'interroger sur la catégorie de la nationalité. En effet, l'importance de l'information tient à sa relative originalité. Il est possible de remarquer que la nationalité ne figure pas dans la plupart des autres sources d'origine professionnelle ou économique. Ainsi les registres des patentes, impôts des travailleurs indépendants mis en place au XIX ${ }^{c}$ siècle. ne mentionnent pas la nationalité dans l'entredeux-guerres. Quelles sont donc les raisons qui poussent à la différenciation nationale, au 
sein d'un document économique? Il est important de décrire les modèles idéologiques qui šinsinuent derrière les catégories statistiques. Car les catégories du Registre du commerce ont une histoire.

\section{L'histoire du Registre du commerce}

\section{La création du Registre: identifier l'étranger}

Le Registre du commerce est institué en France par la loi du 18 mars 1919. Avant cette date. lors de l'ouverture d'un commerce, les formalités consistaient seulement dans le dépôt d'un double de l'acte de société, au greffe du Tribunal de commerce du lieu de l'établissement. Les commerçants n'optant pas pour la forme juridique d'une société se trouvaient ainsi affranchis de toute déclaration officielle. Dès le début du siècle, plusicurs voix se font entendre en faveur de la création d'un Registre du commerce ${ }^{16}$. Mais ce n'est que pendant la guerre de 1914-1918 que le débat franchit le seuil de l'Assemblée avec le dépôt. le 17 octobre 1916, d'une proposition de loi allant dans ce sens. L'exposé des motifs de la loi explique que le Registre du commerce a pour objet d'organiser ce que l'on pourrait appeler "l'État civil des établissements commerciaux» et qu un tel document comblerait les lacunes de la législation alors en vigueur où la publicité faite à tous les actes de la vie commerciale est tellement éparse que les divers intéressés rencontrent de grandes difficultés à se procurer les renseignements dont ils ont besoin: "Qu il s"agisse, en effet, de connaître la nationalité d'un commerçant, le régime juridique sous lequel il est marié, les établissements qu iil a dirigés précédemment ou qu il exploite simultanément en France ou à l'étranger, la publicité telle qu'elle est organisée actuellement présente de fâcheuses lacunes ou une dispersion regrettable $»^{17}$. Notons que l'argumentation réserve d'emblée une grande place à la question de la nationalité du commerçant, qui vient au premier rang des informations utiles à connaitre.

En effet, le contexte de la première guerre mondiale, moment important bien que mal connu de rejet des étrangers ${ }^{18}$, imprime sa marque sur les motifs légitimant la création d'un Registre de commerce qui, selon les termes du projet de loi, "présente au point de vue de la défense de notre industrie et de notre commerce nationaux contre l'emprise de l'activité économique étrangère, des conséquences particulièrement heureuses. Des établissements étrangers qui, comme on l'a constaté trop souvent, se donnaient facilement une apparence d'entreprise française, seront désormais tenus de fournir tous les renseignements de nature à fixer le public sur leur véritable nationalité ${ }^{19}$. L'argument de la nationalité est encore ici premier dans les justifications des législateurs. La première guerre mondiale exacerbe la logique de différenciation nationale, à l'cuuvre depuis les débuts de la III ${ }^{e}$ République, puisque la défense de la patric semble passer par la "protection " du marché du travail contre les étrangers. Le commerce apparaît comme un objet privilégié des discours qui arguent alors d'un «danger du point de vue national». "Il est nécessaire de voter des lois qui s'imposent immédiatement. Ce sont celles qui ont pour but de barrer la route à l'invasion dans notre pays de nos ennemis qui, tout en préparant la guerre qu ils préméditaient depuis de nombreuses années, s'installaient peu à peu chez nous pour ouvrir nos villes aux hordes des barbares" peut-on ainsi lire, dès le 22 décembre 1914, dans le texte d'une proposition de loi visant à interdire à tout individu d'origine allemande de posséder des propriétés $\mathrm{s}^{20}$. De plus, la mobilisation sur le front des commerçants français alimente les craintes face à la concurrence des étrangers, accusés d'en profiter pour élargir leurs clientèles. Jamais invoqué directement dans les textes officiels, l'argument de la "concurrence 
21. ACCIP cote III. 4.44 (1).

22. On remarque d'ailleurs durant l'entre-deux-guerres la sous-représentation des étrangers dans le secteur «Vins, Bars et Brasseries".

23. Rapport de la Chambre de commerce de Paris sur le projet de loi visant à instituer un registre de commerce, 16 décembre 1916, ACCIP cote III. 3.20 (3).

24. Ernest Gellner cité par Gérard Noiriel, Population, immigration et identité nationale en France, $X I X^{e}-X X^{e}$ siècle, Paris, Hachette, 1992, p. 114.

25. Rapport anonyme de la Chambre de commerce de Paris sur les naturalisations des commerçants étrangers, janvier 1915. ACCIP cote III. 4.44 (4).

26. Raoul de Grasserie, «De l'institution du Registre de commerce ", Revue du Commerce et de l'Industrie, $2^{\mathrm{e}}$ année, $n^{\circ} 9$, septembre 1895 , p. 337.

27. Nonna Mayer, La boutique contre la gauche, Paris, Presses de la Fondation nationale des sciences politiques, 1986, p. 101. déloyale » figure quasi-systématiquement dans les pièces des dossiers des Chambres de commerce relatifs au Registre du commerce comme, par exemple, dans la lettre d'un voyageur de commerce lyonnais adressée au Président de la Chambre de commerce de Paris, le 25 septembre 1916: «Pendant que ces bons Français se font tuer pour cinq sous par jour, il y a en France des quantités de marchands espagnols, italiens, suisses etc... qui naturalisés ou non, et grâce au prix élevé des produits, se font de bonnes et solides rentes à nos dépens $" 21$. Ces plaintes trouveront un écho dans l'appareil législatif mis en place pendant la guerre comme l'illustre la loi du 9 décembre 1915 qui établit qu'à moins de posséder une résidence de cinq ans en France, les étrangers ne peuvent ouvrir un débit de boissons ${ }^{22}$.

Il faut ainsi inscrire la création du Registre de commerce dans un contexte particulier d'exacerbation de la logique de différenciation nationale et il est intéressant de noter que l'institution du Registre est légitimée, dans le discours, par le «problème des étrangers». Les débats autour de la création d'un Registre du commerce évoquent tous la nécessité de protéger le commerce français contre «l'envahissement étranger». Il est d'ailleurs possible de préciser que si le Registre du commerce n'est pas une création française - il existe dans plusieurs autres pays en 1914, et en particulier en Italie, en Espagne, dans les pays scandinaves, en Allemagne mais aussi dans quelques pays d'Amérique latine comme l'Argentine et le Chili - la France innove en demandant qu'y figure la nationalité. Il s'agit d'une nouveauté par rapport aux autres Registres du commerce mis en place et les auteurs de cette proposition s'en félicitent à plusieurs reprises, rejoints par les experts chargés d'étudier le projet de loi: «Aucune autre nation jusqu'à ce jour ne nous semble avoir imposé cette indication [...] s'il avait existé longtemps avant la guerre, [ce Registre] nous eût peut-être évité la surprise d'un certain envahissement de 
notre marché par les étrangers avides de mettre la main et d'imposer leur hégémonie sur notre commerce et notre industrie ${ }^{23}$.

Il est alors possible d'analyser l'institution du Registre de commerce comme un moment de la «révolution identitaire» qui met progressivement fin aux modalités d'identification propres à la logique préindustrielle où l'interconnaissance des individus au sein de leur communauté locale permettait l'identification directe. Au début du XIX ${ }^{\mathrm{c}}$ siècle, le critère de la résidence vient encore en premier dans la définition de soi. À partir du moment où de plus en plus de droits, civiques mais aussi sociaux, dépendent du critère de la nationalité, apparaît le besoin de documents officiels chargés d'authentifier: les papiers d'identité. La nationalité devient un élément fondamental de l'identité personnelle: «Un homme doit avoir une nationalité comme il doit avoir un nez et deux oreilles ${ }^{24}$. Il faut donc l'afficher, la rendre publique. Les cartes d'identité d'étrangers sont obligatoires à partir de 1917 et un certain nombre de suggestions sont émises, concernant la nationalité des commerçants. Ainsi de l'idée d'un tableau de nationalité qui naît lors des débats relatifs aux cartes d'identité des commerçants étrangers en 1915: «Établi à des dimensions invariables, de 35 à $40 \mathrm{~cm}$ sur 25 à $30 \mathrm{~cm}$, ce tableau sera exigible pour tout commerçant, industriel, fonctionnaire, avocat, médecin, journaliste, etc., ayant affaire au public dans l'exercice de sa profession ou fonction et devra être placé d'une manière apparente dans le bureau, magasin, atelier du titulaire, dans la partie du local où il reçoit ordinairement le public. Grâce à cette amplification voulue de la carte de nationalité il sera possible d'y écrire ou imprimer certaines mentions essentielles de manière qu'elles puissent être lues d'un seul coup d'œil, autrement dit qu'elles soient mises à la portée de tous. Des lettres de un ou deux centimètres de hauteur, d'un caractère approprié, permettront de lire aisément le nom du lieu, le pays d'origine, et la nationalité, puis, pour le cas d'un naturalisé français, la date de naturalisation et la localité où a été établi l'acte. ${ }^{25}$. La proposition d'un tableau de nationalité ne sera pas suivic d'effet mais elle illustre l'importance de la catégorie de "nationalité" dans la mise en place des processus d'identification des commerçants pendant la guerre de 1914-1918, processus qui aboutissent à création du Registre du commerce. L'identité doit être affichée, rationalisée, catégorisée.

Mais au delà du seul critère de la nationalité, le Registre du commerce dans son ensemble peut être analysé comme l'illustration de l'intervention croissante des pouvoirs publics dans la codification de l'identité personnelle. L'ingérence de l'État est cependant souvent mal perçue dans le secteur commercial où la discrétion et le secret comptent parmi les revendications traditionnelles $\mathrm{du}$ commerçant. Dès la fin du XIX ${ }^{\mathrm{e}}$ siècle, le projet de Registre du commerce provoque des débats dans les milieux juridiques et commerciaux qui se font souvent l'écho de ces réticences. Ainsi de ce juge au Tribunal de commerce de Rennes qui s'inquiète dès 1895: « Ne vaut-il pas mieux laisser chaque commerçant exercer son commerce, comme il le fait aujourd'hui, hors du regard intéressé et souvent hostile des tiers? Est-ce que le secret des opérations n'est pas essentiel au commerçant le plus honnête? ${ }^{26}$. La mise en place du Registre du commerce ne peut manquer de provoquer certaines tensions parmi les petits commerçants qui commencent alors à être représentés politiquement et glissent progressivement vers la droite, comme l'a montré Nonna Mayer ${ }^{27}$. Ainsi l'une des toutes premières organisations professionnelles, la Ligue syndicale pour la défense des intérêts du travail, de l'industrie et du commerce créée en 1888 , fédère les détaillants autour de deux principes: la défense de l'indépendance économique et le combat contre la centralisation 


\section{$\rightarrow$}

28. Philip Nord. "Le mouvement des petits commerçants et la politique en France de 1888 à 1914 », Le Mouvement social, 1981, n²114, p. 37.

29. Voir Gérard Noiriel, «L'identification des citoyens. Naissance de l'état-civil républicain ", Genèses, $n^{\circ} 13$, 1993. pp. 3-28.

30. Notons que leur budget est alimenté par une contribution additionnelle sur la patente.

31. Lettre d'Auguste Isaac, ministre du Commerce et de l'Industrie en date du 30 novembre 1920, ACCIP cote III.3.20 (3).

32. Rapport de l'Union des Chambres syndicales lyonnaises du 16 juin 1921, ou encore Communication de la Chambre syndicale patronale des fabricants d'accessoires et pièces détachées d'automobile, de cycles et d'appareils aćriens le 23 juillet 1921. ACCIP cote III.3.20 (3).

33. Voir l’approche quantifiée du phénomène dans « Les étrangers et les naturalisés dans la société française. Commentaire des recensements de 1931 et 1936" in Eric Guichard et Gérard Noiriel (dir.), Construction des nationalités et immigration dans la France contemporaine, Paris, Presses de l'École normale supérieure, 1997, pp. 39 et suiv.

34. Ralph Schor, L'Opinion française face aux étrangers, 1919-1939, Paris, Publications de la Sorbonne, 1985.

35. «Protection du commerce honnête», Rapport de la Chambre de commerce de Châteauroux et de l'Indre, 11 avril 1933, ACCIP cote III. 4.44 (2).

36. En majuscules dans le texte.

37. ACCIP, cote III. 4.44 (2). étatique, comme l'explique Philip Nord: « Pour la Ligue, l'ennemi comprenait [...] l'État moderne, l'État bureaucratique et interventionniste ${ }^{28}$. Aux yeux des commerçants, le Registre du commerce incarne un moyen de contrôle étatique. L'expression utilisée officiellement pour le décrire, "état-civil des établissements commerciaux», est fort explicite et nous rappelle les problèmes rencontrés au début du XIX ${ }^{\mathfrak{e}}$ siècle lors de l'instauration de l'état-civil en France ${ }^{29}$. De plus les réticences de la profession sont exprimées au moyen de relais institutionnels qui semblent constituer de véritables groupes de pression au tournant du siècle. Outre les différents syndicats professionnels (Chambres syndicales, Ligues professionnelles, etc.), les Chambres de commerce constituent des organes intermédiaires entre les institutions syndicales et les pouvoirs publics: leur avis doit être pris en compte par l'administration avant toute réglementation des usages commerciaux et préalablement à diverses mesures touchant le commerce ${ }^{30}$. Les archives révèlent de nombreuses traces témoignant de l'intervention des Chambres de commerce auprès des députés et du ministère du Commerce. Les premiers projets de registres de commerce sont ainsi successivement enterrés, en 1895 puis en 1901.

Quelques vingt-cinq ans plus tard, lors de la création du Registre, la méfiance continue de dominer comme l'indiquent les faibles taux d'immatriculation lors des premiers mois qui suivent la mise en place du Registre du département de la Seine: 350 immatriculations par jour en octobre 1920 , soit trois mois après l'ouverture, contre plus de 1600 en décembre de la même année. Les pouvoirs publics, par la voix du ministre du Commerce et de l'Industrie, s'inquiètent des réticences face au Registre et multiplient les rappels de l'obligation d'immatriculation par voie d'affiches, communiqués aux journaux et annonces diverses. Un avis destiné à être affiché est ainsi transmis aux Chambres de commerce qui rap- 
pelle les sanctions en cas de défaut de déclaration $^{31}$. Malgré les mises en garde, le délai d'inscription doit être prolongé de six mois par la loi du 30 décembre 1920. Cependant cela ne suffit pas. Les pouvoirs publics sont contraints de renforcer l'appareil législatif en rendant obligatoire le numéro d'immatriculation au Registre sur tous les imprimés et papiers de commerce (loi du $1^{\text {er }}$ juin 1923) mais le texte de loi ne suit pas les recommandations de certains syndicats qui, dès 1921, réclament que soit inscrite sur les papiers de commerce, à côté du numéro d'immatriculation au Registre, la nationalité du commerçant ${ }^{32}$.

Le contrôle des commerçants étrangers apparaît donc comme le principal alibi idéologique qui vient légitimer les processus d'identification dans les discours des pouvoirs publics comme des institutions représentant la profession. Or la différenciation nationale par le biais de la création du Registre, loin de clore les débats, ouvre la voie à d'autres revendications. Ainsi, au sein des débats de l'entre-deux-guerres qui portent sur les modifications éventuelles à apporter au Registre du commerce, la question des étrangers tient un rôle de premier plan.

\section{Les années trente: distinguer le vrai du faux}

Toutes les propositions de modifications du Registre du commerce ne concernent pas les étrangers. Néanmoins, la question occupe une place croissante dans les débats relatifs au Registre du commerce pendant l'entredeux-guerres et surtout dans les années trente. La crise économique engendre une relative stagnation de la population étrangère qui rencontre de plus en plus de difficultés à trouver du travail. Certains expliquent d'ailleurs la faiblesse relative des taux de chômage français comme une conséquence de la structure particulière du marché du travail en France où la population industrielle est forte d'une main d'œuvre étrangère plus flexible et moins revendicative. À l'heure de la crise, les travailleurs immigrés rentrent dans leur pays d'origine, et ne "comptent" pas alors comme chômeurs ${ }^{33}$. De plus les restrictions législatives concernant l'emploi d'une main-d'œuvre étrangère ou, pour reprendre la terminologie de l'époque, les lois et décrets de «protection de la maind'œuvre nationale» mettent un frein aux flux migratoires à destination de la France.

D'autre part, la crise fournit le prétexte au développement de courants xénophobes ${ }^{34}$. Les textes de source professionnelle se font ainsi souvent durs, parfois virulents contre les petits commerçants étrangers. La rhétorique se fonde alors sur l'obsession de la dissimulation de l'origine. L'argumentaire qui, hier, vantait les mérites de la discrétion et du secret pour la bonne tenue d'un commerce, change radicalement. Pour "protéger le commerce honnête", il devient désormais nécessaire de «démasquer l'étranger». La Chambre de commerce de Châteauroux et de l'Indre se plaint ainsi de la «mentalité déplorable» de certains étrangers: "Le commerce et l'industrie en général, et principalement dans la maroquinerie et la fabrication des vêtements de cuir [...], sont la proie d'inconnus, de réfugiés venant d'on ne sait où. Il est commun de trouver ces indésirables sous le couvert de noms de fantaisie, de sociétés douteuses, masquant leur véritable origine $»^{35}$. La logique d'identification fait place à la crainte de la falsification et le problème du faux devient central. «Nous luttons désespérément contre l'envahissement incessant de FAUX ARTISANS ÉTRANGERS ${ }^{36}$ dans notre profession» déclare l'Association parisienne des fabricants de chapeaux pour dames, le 13 juin 1935, dans un rapport transmis par la Chambre de commerce de Paris à Pierre Laval, alors Président du Conseil $1^{37}$. Le débat dépasse alors les cadres professionnels et prend place dans la sphère politique illustrant par là la perméabilité des frontières entre les deux mondes économique et politique, per- 
38. Marie-Claire Laval-Revigiio, «Parlementaires xénophobes et antisémites sous la III' République. Débats relatifs à la nationalité et à la "protection du travail national" ", Le droit antisémite de Vichy..., op. cil., pp. 85114.

39. Denais fait référence à la loi du 19 juillet 1934 selon laquelle, pendant dix ans à partir du décret de naturalisation, l'étranger naturalisé ne peut être nommé à des fonctions publiques rétribućes par l'État, inscrit à un barreau ou nommé titulaire d'un office ministériel.

40. Proposition de loi n" 4699 déposée à la Chambre des députés le 12 février 1935, Journal officiel, Documents parlementaires, p. 1434.

41. Ainsi des propositions de loi de MM. Lachal (Chambre des députés, $n^{\prime \prime} 2494,10$ novembre 1933). Deudon et Grisoni (Chambre des députés, $n^{\circ} 2704,15$ décembre 1933), Amidieu du Clos (Chambre des députés, $\mathrm{n}^{\circ} 1813.13$ avril 1934).

42. Proposition de loi n" 4699.... op. cit.

43. Voir Gérard Noiriel, La Tyrannie du national. Le droit d'asile en Europe (1793-J993), Paris, Calmann-Lévy, 1991. méabilité qu'illustre l'expression employée par Marcel Bagnaud: «L'État, c'est nous».

La xénophobie franchit sans peine l'enceinte du Parlement français ${ }^{38}$. Un certain nombre de propositions de loi font écho aux requêtes des institutions professionnelles. Et il est intéressant de noter, à ce propos, les mécanismes qui permettent le passage d'une logique identifiante à une logique discriminante. En effet, le Registre du commerce semble s'imposer naturellement comme le moyen non plus d'identifier mais de contrôler, voire d'interdire l'activité commerciale aux étrangers. Quoi de plus facile que de passer d'un état civil commercial à un "casier commercial» selon l'expression du député de la Seine, Joseph Denais qui propose, en 1935, d'instituer une nouvelle réglementation lors de l'inscription au Registre du commerce: "N'importe qui, venant de n'importe où, peut tenir en France commerce de n'importe quoi. Il a paru nécessaire de réglementer la main d'œuvre étrangère. Il est devenu indispensable d'interdire aux étrangers, même pendant les premières années qui suivent leur naturalisation, l'exercice de certaines professions libérales et si on ne l'a pas encore fait pour les médecins, on l'a fait pour les avocats et les professeurs de l'Université ${ }^{39}$. Le député, membre de la Fédération républicaine du très conservateur Louis Marin, invite ainsi à suivre la voie ouverte par «la plupart des pays voisins [qui] soumet l'exercice des professions commerciales à des règles strictes, notamment en ce qui concerne les étrangers » et n'hésite pas à citer la législation nazie en exemple, puisqu'il fait référence à la loi allemande du 12 mai 1933 qui interdit de créer de nouveaux magasins de détail. Denais propose donc à la Chambre des députés une loi selon laquelle un délai de cinq années de résidence continue en France devrait être exigé pour obtenir une inscription au Registre du commerce ${ }^{40}$. Ce dernier devient dès lors 
l'instrument potentiel d'une discrimination sur le marché du travail.

Et ce texte n'est pas isolé: en visant «la défense du commerce français ", diverses propositions de loi revendiquent l'obligation d'une autorisation administrative à l'établissement d'un commerçant étranger ${ }^{41}$. D'autres réclament qu'il soit fait plus clairement mention de la nationalité des commerçants en proposant, par exemple, qu'en toutes circonstances où il est tenu de faire mention de son inscription au Registre du commerce, le commerçant soit contraint d'indiquer sa nationalité «de la même façon et dans les mêmes caractères ${ }^{42}$. Ils suggèrent même que nul établissement ne puisse être ouvert au public s'il ne porte sur son accès principal mention du nom du propriétaire ou de l'administrateur délégué, «avec mention, en toutes lettres, de leur nationalité». Inscrire la nationalité du commerçant à l'extérieur de la boutique, nous ne sommes plus loin du «tableau de nationalité » réclamé par certains en 1915. En insistant sur la prétendue «moralité douteuse » des commerçants étrangers, certains députés requièrent des enquêtes sur leurs éventuels antécédents judiciaires, leurs ressources financières, etc.

Il est intéressant de remarquer que ces propositions de loi ne sont pas l'apanage exclusif de la droite parlementaire: sur les vingt-deux députés ayant proposé à la Chambre ou au Sénat des textes législatifs visant à limiter l'exercice du commerce par les étrangers dans les années trente, on compte quatre membres du Parti radical, quatre socialistes et même un dissident du Parti communiste. Certes, jusqu'en 1938, aucune des propositions concernant spécifiquement les commerçants étrangers n'est adoptée. Mais si elles n'ont pas d'effet législatif, ces propositions de modification de la législation sur le Registre du commerce témoignent d'une rhétorique qui fait de la dissimulation de l'origine un problème cen- tral et elles illustrent l'obsession, croissante dans les années trente, de rendre la nationalité visible, obsession sous-tendue par les thèmes de la lutte contre la clandestinité et de la chasse aux dissimulateurs qui se développent en créant un climat de suspicion permanente sur le statut des étrangers. Les difficultés à faire reconnaître le statut de réfugié pour les émigrés italiens, allemands et espagnols dans l'entre-deux-guerres alimentent la défiance systématique à l'égard des étrangers, défiance qui se transforme fréquemment en xénophobie ${ }^{43}$.

Et si aucune des propositions de loi mentionnées plus haut n'est acceptée, on constate dès 1935 un durcissement par voie réglementaire de la législation à l'égard des indépendants étrangers. Les manifestations de rejet des commerçants étrangers, révélatrices d'une xénophobie croissante de la société française, ne restent pas cantonnées à la sphère des discours. En effet, le secteur des indépendants n'est pas épargné par les mesures de "protection de la main d'œuvre nationale»: le décret du 2 août 1935 institue une carte d'artisan étranger qui permet de fixer la proportion d'artisans étrangers par industrie et par région. Le 30 octobre 1935, un autre décret subordonne le commerce ambulant étranger à une déclaration à la Préfecture: aucune autorisation ne peut être accordée à un étranger qui ne justifie pas de cinq années de résidence continue en France. Enfin, l'institution d'une «carte de commerçant étranger» par le décret-loi du 12 novembre 1938, vient répondre aux revendications étudiées plus haut.

\section{8-1939: Discriminer}

Le décret-loi stipule qu'il est interdit à tout étranger d'exercer sur le territoire français une profession commerciale ou industrielle sans justifier de posséder une carte d'identité spéciale portant la mention commerçant et délivrée par le préfet du département où 


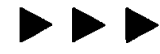

44. Jo, Lois el décrets 1938, pp. 12923 et 13958 et Jo, Lois et décrets 19.39. p. 1645. ('es dispositions se maintiennent bien après la fin de la guerre. Voir ACCIP cote III. $4.44(4)$.

45. Proces-verbal de la séance du 23 novembre 1938 consacréc à l'examen du rapport sur La situation des étrangers en France. AC C'IP cote III. 4.44 (2).

46. Proposition de loi n 5417. Chambre des députés. 14 mars 1939. Jo, Documents parlementaires. p. 941.

47. Proposition de loi n" 5769. Chambre des députés. 2 juin 1939. Jo Documents parlementaires. p. 1858. l'étranger exerce son activité. Une circulaire datéc du 28 mars 1939 précise les modalités d'obtention de la carte: la demande s'effectue à la préfecture, puis elle est transmise à la Chambre de commerce du département pour cnquêtes et avis. Les instances professionnelles ne disposent que d'un pouvoir consultatif, mais participent au processus. La décision revient en dernicr lieu au préfet ${ }^{44}$. La mesure répond alors aux deux thèmes majeurs des débats des années trente étudiés plus haut, puisque, d'une part, elle institue une autorisation administrative obligatoire pour exercer l'activité commerciale et, d'autre part, elle renforce les modalités d'identification par la nationalité. Mettre en place une carte d'identité de commerçant étranger revient à institutionnaliser l'identité de commerçant étranger en lui donnant une forme juridique et matérielle. Surtout, la carte, en instaurant le contrôle administratif de l'établissement d'un commerçant, limite grandement la liberté d'ouvrir une boutique pour un étranger.

Et pourtant l'institution d'une carte de commerçant étranger ne met pas fin aux débats. Au contraire, à l'Assemblée comme dans les Chambres de commerce, la question prend encore plus d'importance dans les années 1938-1939 tout en changeant d'objet. La revendication qui s'exprime alors concerne les noms des commerces et des commerçants. Plusieurs rapports des Chambres de commerce expriment le vœu qu'il devienne obligatoire, pour les commerçants et les artisans étrangers établis en France, d'exercer leur profession sous leur nom patronymique. Les arguments évoqués se réfèrent encore ici à une logique commerciale de "concurrence déloyale» comme l'explique en 1938 le rédacteur d'un rapport sur le sujet à la Chambre de commerce de Paris: «Prenons un exemple... Voici la maison Antoine (je prends ce prénom au hasard et ne veux désigner personne). $\mathrm{Ce}$ commerçant a une boutique qui ne se distingue en rien de l'une des nôtres, bien qu'il 
soit Argentin ou Roumain. Une cliente, trompée par les apparences, pénètre chez lui et ne s'aperçoit de son erreur que lorsqu'il est trop $\operatorname{tard} »^{45}$. Le 14 mars 1939, le débat franchit le seuil de la Chambre des députés lorsqu une proposition de loi requiert l'obligation de faire figurer le nom patronymique intégral sur les enseignes et les papiers commerciaux de l'entreprise; elle prévoit que l'usage d'un faux patronyme ou d'un patronyme incomplet soit puni des "peines prévues en matière de faux et d'usage de faux $»^{46}$. Et à peine trois mois plus tard, il s'agit de limiter la faculté, pour les commerçants naturalisés, de modifier leur nom patronymique pendant dix ans, toujours au nom de la protection du consommateur: "Il est inadmissible que des individus n'ayant aucune attache avec le pays qui leur accorde généreusement la nationalité française aient, au surplus, le droit de s'arroger un nom essentiellement français ${ }^{47}$. Là encore, il ne s'agit que de propositions de lois qui n'ont pas donné lieu à l'adoption de textes législatifs. Il peut donc paraître abusif d'en détailler la rhétorique. Pourtant je les cite ici car elles font sens: les débats qui ont lieu à la Chambre en 1938 et 1939 montrent que l'information autour du nom du commerce n'est pas neutre et qu'elle engendre une assignation identitaire. C'est pourquoi la question du nom du commerce permet d'aborder la relation existant entre les débats publics et les individus concernés par ces débats.

En effet, l'étude de l'histoire du Registre du commerce et des débats sur la législation relative aux commerçants étrangers peut paraittre, à première vue, éloignée de l'histoire des commerçants eux-mêmes. Le cloisonnement des différents champs historiques a longtemps contribué à séparer les domaines de recherche réservant l'étude de la législation et des débats institutionnels aux historiens du politique alors que l'historien du social devait se cantonner à l'exploitation de sources concernant directement les groupes sociaux, en particulier des sources quantitatives. Mais il semble nécessaire de tenter de dépasser ces oppositions pour prendre en compte les effets des textes législatifs sur les modalités de liidentité sociale puis personnelle des individus. Est-il possible. à partir de méthodes quantitatives. de mettre en lumière les liens qui existent entre les pratiques étatiques et les stratégies identitaires du commerçant étranger?

\section{La question du nom du commerce}

Les débats qui ont lieu à la Chambre en 1938 autour des noms des établissements tenus par des étrangers ou des Français naturalisés montrent que l'information n'est pas anodine. Les étrangers sont accusés de cacher leur origine en dissimulant leur nom. Or ces controverses trouvent une illustration avec le cas de Norbert Elias, évoqué plus haut, qui ouvre sa boutique en 1934 en la nommant d'après son prénom: les «Ateliers Norbert». L'information peut fournir matière à l'anecdote, mais qu'en dire de plus? Les renseignements sur les noms semblent en effet constituer des données a priori difficilement exploitables statistiquement. Constitutif de l'individualité, le nom se soustrait aux procédures d'agrégation, nécessaires aux fins de produire une statistique: comment coder un nom? De plus, les noms de commerce sont dans leur grande majorité (près de $70 \%$ ) identiques aux noms des commerçants. Malgré ces difficultés, l'idée est née de faire du nom du commerce un indicateur statistique d'une stratégie face à la stigmatisation. L'hypothèse de recherche selon laquelle, afin d'échapper à la stigmatisation, les étrangers utiliseraient plus volontiers des noms de commerce différents de leurs patronymes, se révèle pourtant fausse. Selon un sondage effectué aléatoirement sur $250 \%$ immatriculations au Registre du commerce entre 1920 et 1939. $69 \%$ des étrangers ouvrent une boutique portant leur patronyme. pourcentage 
strictement identique aux Français. Ce sont les naturalisés qui se distinguent ici, beaucoup moins nombreux à afficher leur patronyme (seulement $50 \%$ mais ce chiffre doit être interprété avec prudence en raison du faible nombre de naturalisés dans l'échantillon, 58 individus uniquement). La différence peut être interprétée comme le signe chez les commerçants naturalisés d'une revendication de leur francité mais peut également traduire une volonté d'échapper aux stigmates de leurs patronymes, reflets d'une origine qui devient souvent honteuse à porter dans la France des années trente. Il est intéressant, à ce propos, de constater que le taux d'adoption d'un nom de commerce distinct du nom patronymique croît tout au long de l'entre-deux-guerres, en particulier chez les naturalisés, évolution qui permet d'approcher l'influence des débats évoqués plus haut sur les individus.

Afin de vérifier cette hypothèse, il semble néanmoins nécessaire de mener une analyse plus fine des noms de commerce. Car le pourcentage de ceux qui choisissent un nom de commerce différent de leur patronyme augmente dans toutes les catégories d'individus, sans distinction de nationalité, au cours de l'entre-deux-guerres. En effet, choisir un nom différent du sien pour désigner une boutique ne signifie pas a priori mettre en place une stratégie de dissimulation d'un «nom étrange» - pour ne pas dire un «nom étranger»-, il peut aussi constituer une méthode commerciale destinée à attirer le client. C'est pourquoi l'indicateur formé par les noms de commerce a dû être affiné et plusieurs catégories ont été distinguées en raisonnant uniquement sur les commerces aux noms différents des patronymes des commerçants (voir tableau p. 117).

La première catégorie comprend l'ensemble des noms de commerce jouant avec le nom du commerçant (à des fins de représentativité statistique, on a regroupé les stratégies de jeu avec le nom et avec le pré- 
nom, mais il sera nécessaire de les distinguer par la suite). Ainsi de Barouyz Andreassian qui pour son atelier de confection mutile son patronyme en choisissant le nom «Maison André", ou de ceux qui préfèrent afficher leur prénom («Boucherie Charles») comme les nombreux propriétaires de débits de boisson qui jouent avec la familiarité associée au prénom («Chez Jean», «Bar Louis») mais aussi Moïse Roisman qui préfère «Maurice » à Moïse. Les commerçants étrangers sont fortement sur-représentés dans cette catégorie (31,7\% contre moins de $15 \%$ chez les Français). Notons cependant que les étrangers sont également sur-représentés dans la deuxième catégorie qui réunit les noms de commerce «régionaux» autrement dit les noms qui font référence à l'origine du commerçant: " $\mathrm{Au}$ Régal Milanais» pour cette épicerie tenue par un Italien, «Au rendez-vous des Suisses» ou encore les établissements qui portent les noms des provinces comme la bonneterie « $\mathrm{A}$ la petite normande» ou la boutique «Photo Lorraine» immatriculées par deux commerçants nés respectivement au Havre et à Nancy. Mais ce sont essentiellement les étrangers qui choi- sissent d'affirmer leur origine sur leur enseigne, sur-représentation qui illustre l'existence d'une stratégie d'affichage publicitaire de l'origine, l'étrangeté devenant alors un atout commercial. Ainsi, sans pouvoir conclure à l'existence d'une stratégie unique face à la stigmatisation, revendication commerciale ou dissimulation, l'indicateur permet de montrer l'importance de l'enjeu identitaire dans le choix d'un nom de commerce chez les étrangers immatriculés au Registre du commerce: les deux premières catégories réunissent près de $38 \%$ des étrangers, contre moins de $20 \%$ des Français.

Ces derniers choisissent pour plus de $65 \%$ des noms de commerce centrés sur l'objet («Salon École de Coiffure», «Charcuterie nouvelle" ou encore "À la bonbonnière", «Cinéma moderne»), sur l'adresse («Librairie de la Poste», «Pharmacie de l'Élysée») ou encore des noms publicitaires («Aux véritables occasions", "Au sans rival») contre $40 \%$ chez les étrangers. Ce bref exemple démontre que les noms de commerce sont plutôt choisis par les Français en fonction des caractéristiques du commerce - objet, lieu... -

\begin{tabular}{|l|c|c|}
\hline \multicolumn{3}{|c|}{$\begin{array}{c}\text { Les noms de commerce différents du patronyme: } \\
\text { répartition selon la nationalité du commerçant (en pourcentages) }\end{array}$} \\
\hline Jeux avec nom & FraNÇAIS & ÉTRANGERS \\
\hline Noms de pays & 16,5 & 31,7 \\
\hline Objets du commerce & 3,2 & 6,1 \\
\hline Indications de lieu & 36,5 & 21,8 \\
\hline Noms publicitaires & 10,2 & 7,1 \\
\hline Associés & 19 & 12 \\
\hline Autres & 7,9 & 13,2 \\
\hline Ensemble & 6,7 & $\mathbf{1 0 0}$ \\
\hline \multirow{2}{*}{ d'immatriculation portant sur un échantillon de 25(K) inscriptions éntre 1920 et 19.39, AVP cote D33U3 } \\
\hline
\end{tabular}


alors que les étrangers privilégient volontiers un rapport à l'identité du commerçant - nom. prénom, origine... Mais ces premières perspectives de recherche mériteront un examen plus approfondi, qui tienne compte en particulier de l'incidence du type de commerce et du quartier de localisation de la boutique sur le nom choisi.

L'indicateur statistique constitué par le nom de commerce permet de faire le lien entre les sources discursives et les sources quantitatives en montrant l'émergence d'un enjeu identitaire associé aux noms et aux désignations. Un choix qui apparaît, dans un premier temps, comme le produit d'une stratégie commerciale relève également d'une logique identitaire. Inversement, il est nécessaire de comprendre comment l'institution du Registre du commerce qui pose la question du nom de l'établissement, différenciée de la question du patronyme du commerçant, détermine les mutations des stratégies économiques des petits commerçants. En officialisant la catégoric "nom de l'établissement " comme un élément d'identification, l'appareil administratif contribue à la diffusion d'une pratique commerciale qui fait du nom un élément de la stratégie publicitaire. L'exemple du nom du commerce montre ainsi les avantages méthodologiques d'une étude conjointe des données et des conditions de leur production. Objet du discours des politiques et des institutions professionnelles, catégorie administrative, le nom du commerce s'impose progressivement pendant l'entre-deux-guerres comme l'un des éléments de la stratégic publicitaire du petit commerce comme en témoigne le pourcentage toujours croissant sur la période des noms de commerce dits publicitaires.

Rappeler combien le travail de l'historien est déterminé par la nature des sources qu'il utilise, revient à énoncer un truisme. L'histoire quantitative construite à partir de sources statistiques produites par les pouvoirs publics a d'ailleurs fait l'objet de critiques qui mettent en valeur les biais introduits par l'utilisation de données codifiées selon des normes spécifiques, parfois même déjà agrégées. C'est ainsi que l'histoire du Registre du commerce, en nous éclairant sur les conditions de production des catégories qui le composent permet de repenser l'exploitation de cette source. L'étude des procédures d'immatriculation tout d'abord a amené à comprendre les modalités de la déclaration d'identité comme une négociation entre le commerçant et les cadres administratifs établis par le formulaire d'inscription. L'analyse des conditions d'établissement du Registre du commerce a ensuite montré les enjeux présidant à la catégorisation en vigueur dans la source. Il s'agit d'identifier les étrangers, clament les législateurs, ce qui permet de légitimer l'intervention de l'État dans un secteur de la vie économique encore réticent à s'intégrer dans les modes dominants de régulation du monde du travail. Mais de l'identification à la discrimination, il n'y a que quelques pas. 